\title{
Magnetism in systems with various dimensionalities: A comparison between Fe and Co
}

\author{
Claude Ederer, ${ }^{1}$ Matej Komelj, ${ }^{2}$ and Manfred Fähnle ${ }^{1, *}$ \\ ${ }^{1}$ Max-Planck Institut für Metallforschung, Heisenbergstrasse 3, D-70569 Stuttgart, Germany \\ ${ }^{2}$ Jožef Stefan Institute, Jamova 39, SI-1000 Ljubljana, Slovenia
}

(Received 17 April 2003; published 6 August 2003)

\begin{abstract}
A systematic $a b$ initio study is performed for the spin and orbital moments and for the validity of the sum rules for X-ray magnetic circular dichroism for Fe systems with various dimensionalities (bulk, Pt-supported monolayers and monatomic wires, free-standing monolayers and monatomic wires). Qualitatively, the results are similar to those for the respective Co systems, with the main difference that for the monatomic Fe wires, the $\left\langle T_{z}\right\rangle$ term in the spin sum rule is much larger than that for the Co wires. The spin and orbital moments induced in the Pt substrate are also discussed.
\end{abstract}

DOI: 10.1103/PhysRevB.68.052402

PACS number(s): 75.30.-m, 75.90.+w

One of the outstanding features of magnetism in lowdimensional spin systems is the importance of the spin-orbitcoupling effects. Whereas the orbital moments in bulk materials of high symmetry are strongly reduced due to orbital quenching, they may attain large values in low-dimensional systems. For instance, in monatomic Co wires at the steps of a vicinal Pt surface an orbital moment of about $0.68 \mu_{\mathrm{B}}$ per Co atom was found, ${ }^{1}$ which is a factor of about 5 larger than in bulk hcp Co and represents the largest orbital moment ever reported for a $3 d$ itinerant electron system. For the magnetic-anisotropy energy of this Co wire, a value was deduced $^{1}$ which is about 50 times larger than the one of hcp Co (which is already large). The large magnetic anisotropy is also relevant for the nature of magnetic excitations. It will generate an excitation gap for the linear excitations, i.e., the spin waves, and for one-dimensional systems it will lead to nonlinear excitation modes which possibly have the character of solitons. ${ }^{2}$

A suitable experimental method to resolve spin and orbital moments is the technique of $\mathrm{x}$-ray magnetic circular dichroism (XMCD). This technique ${ }^{3}$ is based on the fact that for magnetic systems, the absorption coefficient $\mu(\epsilon)$ as a function of the x-ray energy $\epsilon$ is different for $\mathrm{x}$ rays with leftcircular polarization $\mu_{+}(\epsilon)$ and right-circular polarization $\mu_{-}(\epsilon)$. For example, in a $3 d$ transition metal system, one has to measure these absorption coefficients at $L_{2}$ and $L_{3}$ edges corresponding to the $3 d$ transition metal atoms in the investigated system, and then can obtain the orbital moment $m_{l}=-\mu_{\mathrm{B}}\left\langle l_{z}\right\rangle$ and the spin moment $m_{s}=-\mu_{\mathrm{B}}\left\langle\sigma_{z}\right\rangle$ for a transition metal atom via the XMCD sum rules ${ }^{4,5}$ according to

$$
\begin{gathered}
\left\langle l_{z}\right\rangle=\frac{2 I_{\mathrm{m}} N_{\mathrm{h}}}{I_{\mathrm{t}}}, \\
\left\langle\sigma_{z}\right\rangle=\frac{3 I_{\mathrm{s}} N_{\mathrm{h}}}{I_{\mathrm{t}}}-7\left\langle T_{z}\right\rangle, \\
I_{\mathrm{m}}=\int_{E_{\mathrm{F}}}^{E_{\mathrm{c}}}\left[\left(\mu_{\mathrm{c}}\right)_{L_{3}}+\left(\mu_{\mathrm{c}}\right)_{L_{2}}\right] d \epsilon,
\end{gathered}
$$

$$
\begin{gathered}
I_{\mathrm{s}}=\int_{E_{\mathrm{F}}}^{E_{\mathrm{c}}}\left[\left(\mu_{\mathrm{c}}\right)_{L_{3}}-2\left(\mu_{\mathrm{c}}\right)_{L_{2}}\right] d \epsilon, \\
I_{\mathrm{t}}=\int_{E_{\mathrm{F}}}^{E_{\mathrm{c}}}\left[\left(\mu_{\mathrm{t}}\right)_{L_{3}}+\left(\mu_{\mathrm{t}}\right)_{L_{2}}\right] d \epsilon,
\end{gathered}
$$

with the XMCD signal $\mu_{\mathrm{c}}=\mu^{+}-\mu^{-}$and with $\mu_{\mathrm{t}}=\mu^{+}$ $+\mu^{-}+\mu^{0}$. Here, we have assumed that the $z$ axis is parallel to the propagation direction of the $\mathrm{x}$ rays. Then, $\mu_{0}(\epsilon)$ is the absorption coefficient for linear polarization along the $z$ axis. $N_{\mathrm{h}}$ is the number of holes in the $d$ part of the valence band and $\left\langle T_{z}\right\rangle$ is the expectation value of the magnetic dipolar operator

$$
\hat{T}_{z}=\frac{1}{2}[\boldsymbol{\sigma}-3 \hat{\mathbf{r}}(\hat{\mathbf{r}} \cdot \boldsymbol{\sigma})]_{z}
$$

where $\boldsymbol{\sigma}$ denotes the vector of the Pauli matrices. The quantities $E_{\mathrm{F}}$ and $E_{\mathrm{c}}$ denote the Fermi energy and a cutoff energy. For details of such calculations, see Ref. 6.

For a practical application of these sum rules, there are several problems, especially for low-dimensional systems, and we just want to mention two of them. First, the $\left\langle T_{z}\right\rangle$ term, in general, is not known from the experiment, and it is therefore often neglected. However, it is a measure of the anisotropy of the spin density in the material, and it is therefore expected that it becomes more and more important when going to systems with more and more reduced dimensionality and hence more and more reduced symmetry. Stöhr and König $^{7}$ introduced an angle-dependent XMCD technique which allows us to eliminate the $\left\langle T_{z}\right\rangle$ term from the analysis with the spin sum rule. The basic idea is to measure $\mu_{\mathrm{c}}(\epsilon)$ for an orientation of the magnetization in $x, y$, and $z$ directions. For systems with weak spin-orbit coupling, we have $\left\langle T_{x}\right\rangle+\left\langle T_{y}\right\rangle+\left\langle T_{z}\right\rangle \approx 0$, and if this precondition is fulfilled then the contribution of the magnetic dipolar term drops out when we average the spin sum rule over the three directions of the magnetization. However, for low-dimensional systems, the effects of spin-orbit coupling are larger, and it might be that then the precondition for the application of the angle-dependent XMCD is no longer fulfilled. We have shown this explicitly for the extreme situation of monatomic $\mathrm{Fe}, \mathrm{Co}$, and Ni wires. ${ }^{8} \mathrm{~A}$ second problem is that a couple of 

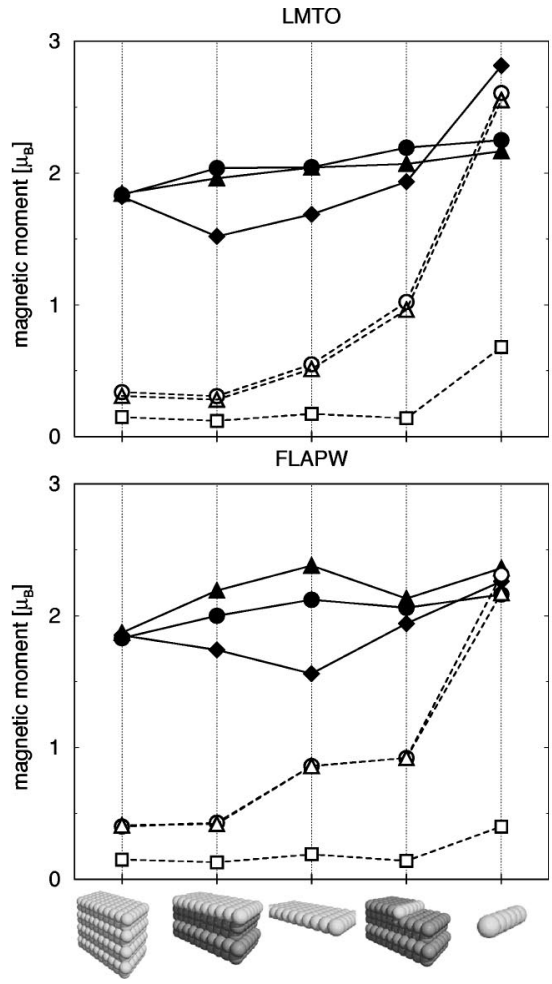
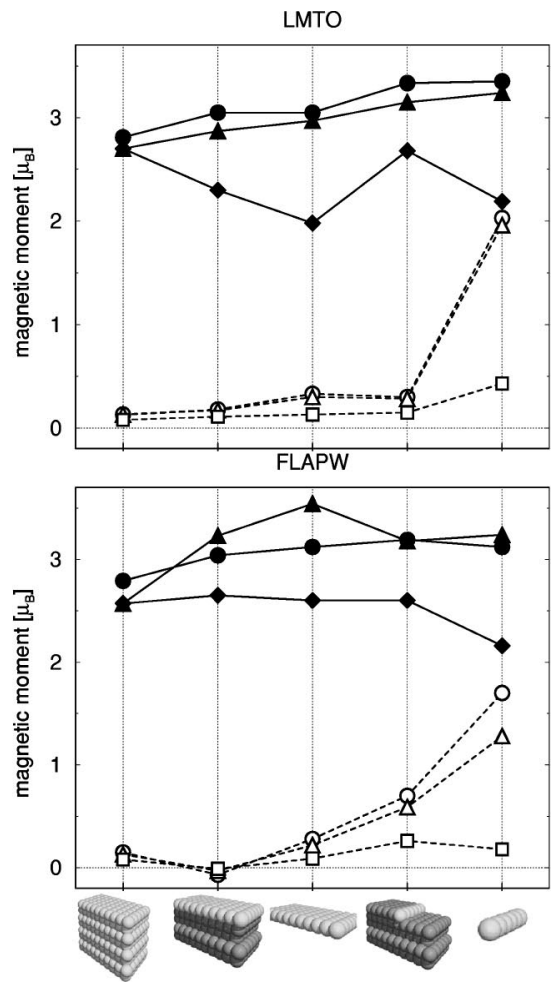

FIG. 1. The results for the spin moments (upper three curves) and for the orbital moments (lower three curves) for Co systems (left part) and for Fe systems (right part) of various dimensionalities. For the meaning of the symbols, see text. The upper graph shows the LMTO results, the lower graph shows the FLAPW results. assumptions and approximations had to be made to derive the XMCD sum rules (see, e.g., Ref. 6, and references therein), the validity of which is not guaranteed in all the systems. The validity of the XMCD sum rules has been confirmed, e.g., for bulk $\mathrm{Fe}$ and $\mathrm{Co},{ }^{9}$ but for atoms at free surfaces ${ }^{10}$ and at interfaces, ${ }^{6}$ i.e., for sites with low symmetry, the application of the sum rules may be critical. It is therefore important to figure out the validity of the sum rules for low-dimensional systems.

In Ref. 11, we performed a systematic ab initio study for the spin and orbital moments and for the validity of the XMCD sum rules in Co systems with various dimensionalities, i.e., for hcp Co, a Co monolayer on Pt (111) and a free-standing Co (111) monolayer, a Co monatomic wire at the steps of a Pt (111) surface as well as for a free-standing monatomic Co wire. In the present brief report, we discuss the respective data for the case of Fe. Corresponding XMCD measurements are on the way for bulk Fe, an Fe monolayer on $\mathrm{Pt}$ and a monatomic Fe wire on $\mathrm{Pt}^{12}$

The validity of the sum rules was tested by calculating the orbital and spin moments on the one hand directly by the $a b$ initio density-functional electron theory; on the other hand, the absorption spectra were determined by the same $a b$ initio theory and then a second set of orbital and spin moments was obtained via the sum rules. Deviations between the two sets of data show that the sum rules are violated. We performed $a b$-initio calculations by using the local-spin-density approximation $^{13}$ (LSDA) in a combination with spin-orbit coupling, without and with an orbital-polarization (OP) term. ${ }^{14}$ The orbital polarization term corrects at least in part explicitely for those electronic correlations which are responsible for the orbital polarization effects (in free atoms described by Hund's second rule) and are not appro- priately described by the LSDA. We used the tightbinding linear-muffin-tin-orbital (LMTO) method in the atomic sphere approximation, ${ }^{15}$ and the WIEN97 code $^{16}$ which adopts the full-potential linearized-augmentedplane-wave (FLAPW) method ${ }^{17}$ in which the spin-orbit coupling and the tools for the calculation of the XMCD spectra $^{18,19}$ and the orbital polarization term ${ }^{20}$ have been implemented. A supercell geometry ${ }^{11}$ was used for all calculations with perpendicular magnetization for the case of the monolayers and wires. For the monolayer, the supercell consists of two $\mathrm{Pt}$ and one Fe (111) layers in the fcc stacking and a vacuum sheet on top of the Fe layer corresponding to two empty layers. The vicinal Pt surface with the Fe wires at the steps is modeled by the supercell shown in Fig. 2. To focus on the pure effect of the dimensionality, we fixed the nearest-neighbor distances of the atoms for all considered systems to the one of fcc Pt $(2.77 \AA)$, as it was done in the LSDA study of finite Co chains on a plane Pt (111) surface by Lazarovits et al. ${ }^{21}$ (In contrast, Spišak and Hafner $^{22}$ have taken into account the structural relaxation effects in their LSDA study of monatomic Fe wires on vicinal $\mathrm{Cu}$ surfaces.)

Figure 1 summarizes the results for the $\mathrm{Co}$ and $\mathrm{Fe}$ systems. For bulk Fe we considered the hypothetical ferromagnetic fcc phase. For the spin moments, nearly the same results were obtained when taking into account the OP term or when neglecting it. We therefore only show the data obtained from calculations with the OP term. Six types of calculations were performed: (1) a direct calculation of the spin moments (filled circles connected by full lines); (2) a calculation of the spin moments based on the spin sum rule, thereby including the $\left\langle T_{z}\right\rangle$ term (filled triangles connected by full lines); (3) a calculation of the spin moments based on the 

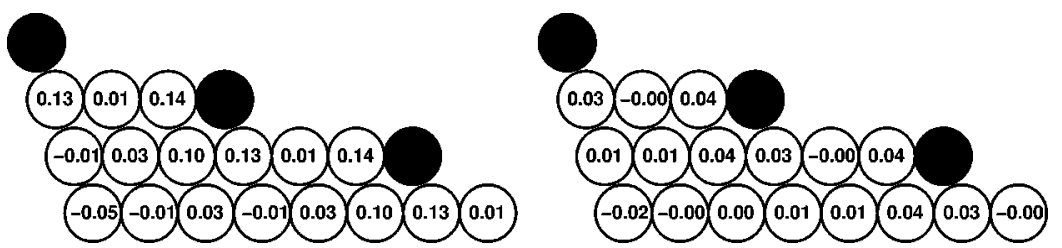

FIG. 2. The spin (upper graph) and orbital (lower graph) moments induced on the Pt atoms (open circles) by the monatomic Fe chains (full circles) at the steps of a vicinal Pt surface. spin sum rule, thereby neglecting the $\left\langle T_{z}\right\rangle$ term (filled diamonds connected by full lines); (4) a direct calculation of the orbital moments in LSDA (open squares connected by dashed lines); (5) a direct calculation of the orbital moments in LSDA + OP (open circles connected by dashed lines); (6) A calculation of the orbital moments in LSDA + OP based on the orbital sum rule (open triangles connected by dashed lines).

The comparison of the results for the LMTO and the FLAPW calculations given in Fig. 1 shows that the general trends are essentially the same for both types of calculations. Quantitatively, there are some differences which result mainly from the fact that the LMTO method adopts a spherical approximation for the effective potential in each atomic sphere (ASA potential), whereas in the FLAPW calculation the full asphericity of the effective potential is taken into account. In the LMTO-ASA method, the $\left\langle T_{z}\right\rangle$ term is determined from the nonspherical charge and spin density obtained after the last iteration step. Because this charge and spin densities are calculated from an ASA potential, the influence of the nonspherical parts of the effective potential within the spheres on the asphericity of the charge and spin densities is neglected. Therefore, for a quantitative discussion, the FLAPW results are more reliable. The most important results are the following.

(a) The spin moments increase only slightly with decreasing dimensionality.

(b) The orbital moments increase strongly with decreasing dimensionality when we take into account the OP term.

(c) For Co, the $\left\langle T_{z}\right\rangle$ term appearing in the spin sum rule is only relevant for the monolayers, otherwise it is rather small (see the more realistic FLAPW data), even for the monatomic wires. It has been outlined in Ref. 11 that this results from the special band filling for the case of Co wires. For Fe, the contribution of the $\left\langle T_{z}\right\rangle$ term to the spin sum rule is considerable for the monatomic layers and also for the monatomic wires. This will represent a problem for the determination of the spin moment from experimental XMCD spectra for Fe monolayers and Fe monatomic wires: The $\left\langle T_{z}\right\rangle$ term cannot be neglected in the spin sum rule, and-at least for the monatomic wires-cannot be determined safely from angle-dependent XMCD measurements because the above discussed precondition for the application of this technique is not fulfilled. ${ }^{8}$

(d) Both the spin sum rule and especially the orbital sum rule are rather well fulfilled for all Co systems, irrespective of the dimensionality. For Fe, there is a tendency that the spin and orbital sum rules are a bit less well fulfilled than for the Co systems, but the violations are still moderate.

When changing the magnetization direction from perpendicular orientation to in-plane orientation (for the monolayer) or in-wire direction (for the chains), the spin moments re- main nearly the same and the orbital moments are only slightly modified so that none of the general statements of points (a) and (b) are affected. The $\left\langle T_{z}\right\rangle$ term depends strongly on the direction of the magnetization, especially for the free-standing wire (see Ref. 8), but the main conclusion that the $\left\langle T_{z}\right\rangle$ term, in general, cannot be neglected in the spin sum rule for systems with low dimensionalities again is not affected.

For an interpretation of the experiments, it is also interesting to know the spin and orbital moments induced by the $\mathrm{Co}$ or Fe atoms in the $\mathrm{Pt}$ substrate, because the Pt atoms contribute to any magnetic property of the system, for instance, to the total magnetization and especially to the magneto-optical properties in the visible regime of light. Experimentally, they can be separated from the spin and orbital moments of the $\mathrm{Co}$ and $\mathrm{Fe}$ atoms by the XMCD technique. ${ }^{3}$ Table I shows the results obtained from the LSDA calculations for the Pt-supported monolayers including the OP term (very similar results are obtained for the pure LSDA calculation). For the Fe monolayer, the induced Pt moments are a bit smaller than for the Co monolayer, especially for the second Pt layer. It should be recalled that no relaxation effects are taken into account and that because of the supercell geometry, there is a vacuum sheet below the second Pt layer. Therefore, the quantitative results should not be taken too literally when comparing with experimental data, but the calculations certainly yield the correct order of magnitude for the polarization effect. A similar discussion holds also for the case of the wires on $\mathrm{Pt}$, for which the results for the monatomic Fe wire obtained by the LMTO method are given in Fig. 2. It is interesting to note that for this geometry, we get nearly exactly the same results as for the case of a monatomic Co wire.

Altogether, the $a b$ initio electron theory does not predict a tremendous difference in the physics of $\mathrm{Co}$ and $\mathrm{Fe}$ monatomic wires although an eventual application of the XMCD spectroscopy on the Fe wires may be less reliable, particularly due to the discussed importance of the magnetic dipolar term.

TABLE I. Results for the induced spin and orbital moments for the Pt- supported monolayers obtained by the LSDA+OP calculations.

\begin{tabular}{lccccccccc}
\hline \hline & \multicolumn{4}{c}{ Co } & \multicolumn{4}{c}{ Fe } \\
& LMTO & FLAPW & LMTO & \multicolumn{2}{c}{ FLAPW } \\
\hline & spin & orbital & spin & orbital & spin & orbital & spin & orbital \\
1. Pt layer & 0.28 & 0.08 & 0.28 & 0.10 & 0.23 & 0.07 & 0.24 & 0.04 \\
2. Pt layer & 0.13 & 0.03 & 0.15 & 0.05 & 0.06 & 0.01 & 0.11 & 0.02 \\
\hline \hline
\end{tabular}


*Electronic address: faehnle@mf.mpg.de; URL: http://physix.mpistuttgart.mpg.de/schuetz/elth/electronth.html

${ }^{1}$ P. Gambardella, A. Dallmeyer, K. Maiti, M.C. Malagoli, W. Eberhardt, K. Kern, and C. Carbone, Nature (London) 416, 301 (2002).

${ }^{2}$ H.J. Mikeska and M. Steiner, Adv. Phys. 40, 191 (1991).

${ }^{3}$ G. Schütz, W. Wagner, W. Wilhelm, P. Kienle, R. Zeller, R. Frahm, and G. Materlik, Phys. Rev. Lett. 58, 737 (1987).

${ }^{4}$ B.T. Thole, P. Carra, F. Sette, and G. van der Laan, Phys. Rev. Lett. 68, 1943 (1992).

${ }^{5}$ P. Carra, B.T. Thole, M. Altarelli, and X. Wang, Phys. Rev. Lett. 70, 694 (1993).

${ }^{6}$ C. Ederer, M. Komelj, M. Fähnle, and G. Schütz, Phys. Rev. B 66, 094413 (2002).

${ }^{7}$ J. Stöhr and H. König, Phys. Rev. Lett. 75, 3748 (1995).

${ }^{8}$ C. Ederer, M. Komelj, J.W. Davenport, and M. Fähnle, J. Electron Spectrosc. Relat. Phenom. 130, 97 (2003).

${ }^{9}$ C.T. Chen, Y.U. Idzerda, H.-J. Lin, N.V. Smith, G. Meigs, E. Chaban, G.H. Ho, E. Pellegrin, and F. Sette, Phys. Rev. Lett. 75, 152 (1995).

${ }^{10}$ R. Wu, D. Wang, and A.J. Freeman, J. Appl. Phys. 75, 5802
(1994).

${ }^{11}$ M. Komelj, C. Ederer, J.W. Davenport, and M. Fähnle, Phys. Rev. B 66, 140407 (2002).

${ }^{12}$ A. Enders and K. Kern (private communication).

${ }^{13}$ J.P. Perdew and Y. Wang, Phys. Rev. B 45, 13244 (1992).

${ }^{14}$ O. Eriksson, M.S.S. Brooks, and B. Johansson, Phys. Rev. B 41, 7311 (1990).

${ }^{15}$ O.K. Andersen and O. Jepsen, Phys. Rev. Lett. 53, 2571 (1984).

${ }^{16}$ P. Blaha, K. Schwarz, P. Sorantin, and S.B. Trickey, Comput. Phys. Commun. 59, 399 (1990).

${ }^{17}$ E. Wimmer, H. Krakauer, M. Weinert, and A.J. Freeman, Phys. Rev. B 24, 864 (1981).

${ }^{18}$ J. Kuně̌, P.M. Oppeneer, H.-C. Mertins, F. Schäfers, A. Gaupp, W. Gudat, and P. Novák, Phys. Rev. B 64, 174417 (2001).

${ }^{19}$ J. Kuněs, P. Novák, M. Diviš, and P.M. Oppeneer, Phys. Rev. B 63, 205111 (2001).

${ }^{20}$ C.O. Rodriguez, M.V. Ganduglia-Pirovano, E.L. Peltzer y Blancá, M. Petersen, and P. Novák, Phys. Rev. B 63, 184413 (2001).

${ }^{21}$ B. Lazarovits, L. Szunyogh, and P. Weinberger, Phys. Rev. B 67, 024415 (2003).

${ }^{22}$ D. Spišak and J. Hafner, Phys. Rev. B 65, 235405 (2002). 